\title{
Étude de la sociologie des exploitants de bois de rose malgaches
}

\author{
Hery F. Randriamalala
}

\section{RÉSUMÉ}

Les exploitants de bois de rose étaient officiellement 13 au début de 2009. Leur nombre est passé à 103 pour la seule région SAVA au début 2012. Mais c'est à Antalaha que leur croissance a été la plus remarquable : les opérateurs historiques ont été rejoints par les membres de leurs familles et leurs anciens collecteurs, puis par des nouveaux venus, pour atteindre le chiffre record de 63 opérateurs dans cette seule ville, malgré I'interdiction d'exploiter le bois de rose depuis mars 2010. Cette communauté a fait d'Antalaha une ville à part à Madagascar car elle y fait jeu égal avec l'État qui n'ose lui appliquer les lois de la République, à moins qu'il n'ait conclu avec elle un mariage d'intérêt. Cette situation s'avère en outre être un invariant historique qui a remarquablement traversé les époques précoloniale, coloniale et postindépendance.

\section{ABSTRACT}

At the start of 2009, there were 13 rosewood traders in Madagascar. By early 2012 their number had increased to 103 traders in the SAVA region alone. It has been in Antalaha that the growth of these traders has been the most explosive: the 'original' traders were joined by family members and also by their former wood collectors. These people were followed by newcomers, the result of which is a current total of 63 traders residing in just this urban centre. Notably, this is despite a ban on the exploiting of rosewood having been implemented in March 2010. Because of the rosewood trading community's ongoing and concentrated activities in Antalaha, this small port could be cited as an urban centre where a faction of the community is actively challenging the State. The State dare not apply the laws implemented towards prohibiting trading of rosewood, but of course, there are common interests between the State and the operators. The current situation described here is not unique: it has recurred through various periods: pre-colonial, colonial and post-independence.

\section{INTRODUCTION}

Il y a exactement 300 ans, en 1713, Carl von Carlowitz, exploitant forestier allemand, inventait le concept d'exploitation forestière durable, ayant pressenti avant tous les autres que les pratiques en cours à son époque allaient mettre en péril l'existence des forêts dans le long terme. Et ce visionnaire avait de bonnes raisons de penser à l'avenir : I'intérêt économique des hommes

\author{
Madagascar \\ E-mail: hery.randriamalala2@gmail.com
}

pour la forêt était déjà sans limite à son époque. Les principales puissances européennes (Hollande, Angleterre, Portugal, Espagne et France principalement) avaient déjà rasé I'essentiel de leurs forêts de bois d'œuvre dès le XVIII ${ }^{e}$ siècle en particulier pour construire les flottes nécessaires à leur commerce international et à sa protection. Très tôt, ces puissances maritimes s'étaient tournées vers I'Amérique du Sud et I'Afrique pour satisfaire leurs besoins en bois imputrescibles, améliorant ainsi la longévité de leurs navires. Le Gabon, le Congo, la Tanzanie, le Mozambique et Madagascar se sont alors mis à vendre ce que les Européens venaient y acheter : bois d'ébène, bois de rose, okoumé, acajou, pour ne citer que les principales essences. À Madagascar, la forêt de Masoala est à la fois exceptionnellement riche et accessible uniquement par la mer. Son exploitation commerciale a donc commencé avant la colonisation. En effet, selon Petit et Jacob (1964), c'est vers 1850 que Hardwick Wilson, négociant mauricien, monte une première scierie au fond de la baie d'Antongil. Ensuite, à partir de 1887, le Premier ministre Rainilaiarivony accorde des concessions à des étrangers pour une exploitation forestière à grande échelle, en raison de services qu'ils ont rendus pendant la guerre franco-merina de 1883 (Petit et Jacob 1964). II s'agit de :

- Désiré Maigrot, créole mauricien : $2500 \mathrm{~km}^{2}$, portés à

7900 en 1891. La concession s'étend d'Antalaha à Cap

Masoala et Maroantsetra ;

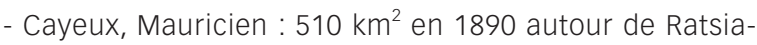
narana ;

- Kingdom, pasteur anglais : $2600 \mathrm{~km}^{2}$ en 1887, de Maroantsetra à Rantabe ;

- Thomé, créole mauricien : $2700 \mathrm{~km}^{2}$ en 1888, de Rantabe à Mananara.

Le pasteur Kingdom était également attributaire en 1889 d'une concession géante d'extraction aurifère, s'étendant de Befandriana à Mandritsara, caché derrière le Gouverneur du Boéni agissant comme prête-nom, moyennant $30 \%$ des revenus extraits de la concession (Decary 1962). À cette époque, les concessions forestières sont soumises par l'État à des clauses financières strictes. L'exploitant doit payer un droit d'exploiter décennal, fournir une part des produits extraits à l'État malgache et verser aux finances publiques un pourcentage de la valeur des produits extraits de la forêt : bois précieux, caoutchouc, gomme copal. Mais déjà, les concessionnaires essaient de se soustraire aux taxes. En 1897, le Résident fran- 


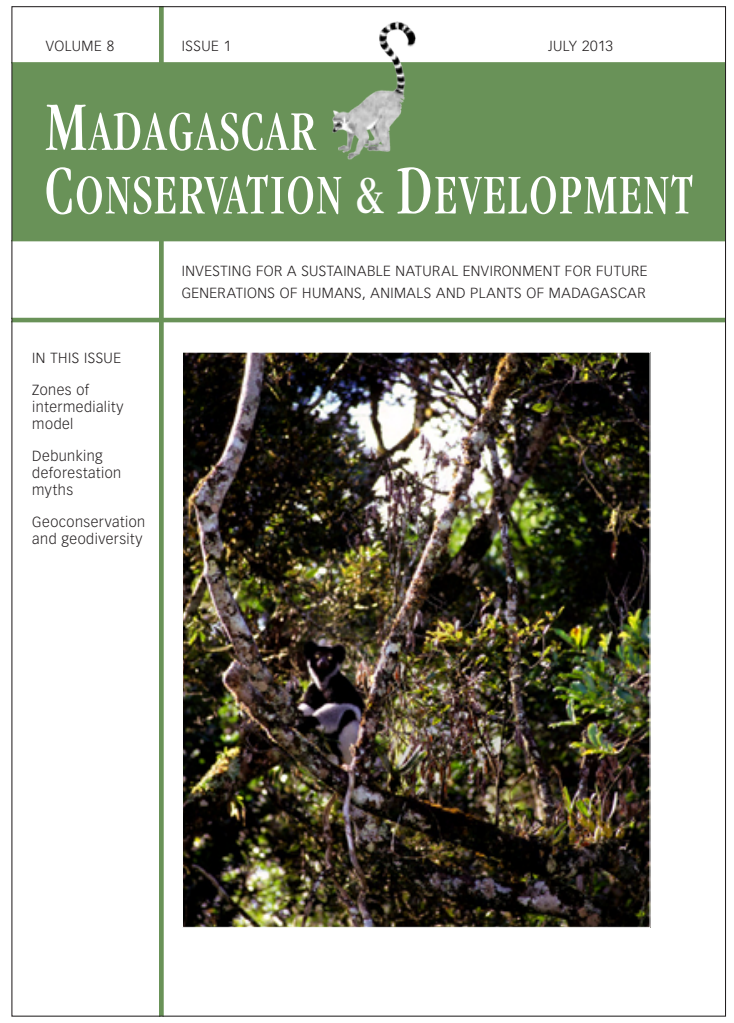

Madagascar Conservation \& Development is the journal of Indian Ocean e-Ink. It is produced under the responsibility of this institution. The views expressed in contributions to MCD are solely those of the authors and not those of the journal editors or the publisher.

All the Issues and articles are freely available at http://www.journalmcd.com

Contact Journal MCD

info@journalmcd.net for general inquiries regarding MCD funding@journalmcd.net to support the journal

Madagascar Conservation \& Development Institute and Museum of Anthropology University of Zurich

Winterthurerstrasse 190

CH-8057 Zurich, Switzerland

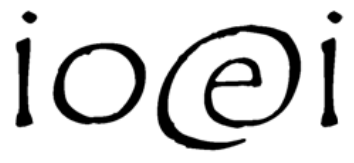

Indian Ocean e-Ink

Promoting African Publishing and Education www.ioeink.com

Missouri Botanical Garden (MBG)

Madagascar Research and Conservation Program BP 3391

Antananarivo, 101, Madagascar 
çais de Vohémar estime que Maigrot fraude de $50 \%$ sur ses extractions (soit 10000 tonneaux de bois et de caoutchouc sortis en fraude sur 10 ans). Ce qui n'empêchera pas Maigrot de faire faillite en 1890, ainsi que son fils en 1896, après avoir repris l'affaire. Le même Résident français de Vohémar rapporte, toujours en 1897, de "véritables faits de brigandage » (Petit et Jacob 1964, p. 42) à I'encontre des autochtones de la part d'un des concessionnaires. L'Administration coloniale ne compte alors que 11 fonctionnaires en 1898 dans cette région d'Antongil, très difficile d'accès. Le commerce du bois est entre les mains de Mauriciens, qui se contentent pour la plupart d'acheter leur production aux villageois, sans organiser eux-mêmes I'exploitation de la forêt. II faut attendre 1904 pour voir arriver les colons français, conformément aux vœux de I'Administration, puis 1908, pour voir s'installer les Chinois dans la région, libérés de la construction du chemin de fer de Toamasina.

En 1899, alors que 30000 hectares étaient en concession autour de Maroantsetra, seuls $20 \%$ étaient réellement exploités, principalement sur la frange côtière en raison du coût de transport des billes provenant de l'intérieur. La fraude sur les bois précieux est alors estimée à $50 \%$ du tonnage déclaré, qui varie de 3000 tonnes en 1910, à 5000 tonnes en 1914 et 1920, les deux meilleures années (Petit et Jacob 1964). Seul Baron Fraise, avec ses trois employés français et sa centaine d'employés malgaches, a installé en 1920 une scierie hautement mécanisée. Les autres exploitants se contentent d'acheter les billes de bois mal dégrossies que leur amènent les villageois, après que ceux-ci les aient difficilement transportées entre le lieu de coupe et le lieu de vente.

Et Petit et Jacob (1964, p. 54) de conclure ainsi I'analyse de cette première phase : "L'exploitation de la forêt de Masoala fut souvent une dévastation à intérêt économique limité. » Les exploitants de cette époque étaient donc des concessionnaires mauriciens, plus aventuriers économiques que créateurs d'entreprise et ils fraudaient l'État à hauteur de $50 \%$.

Au début du $X X^{e}$ siècle, alors que le pouvoir colonial étend son emprise sur l'ensemble de l'île, de nouveaux prédateurs forestiers apparaissent. Les plus gourmands sont proches du pouvoir. II s'agit essentiellement de trois sociétés ayant acquis par convention entre 1902 et 1905 un immense domaine forestier (Fremigacci 1998):

- La société La Grande île, dont le directeur, M. Sisteron, est un ami personnel du gouverneur général Garbit. Cette société a 28000 hectares de forêt, qu'elle défriche par le feu à grande échelle.

- La Compagnie Coloniale. Son directeur, M. Bourdariat, siège au Conseil d'administration de la colonie. Elle a obtenu 17000 hectares de belle forêt grâce à ses appuis politiques à Paris, qu'elle va "exploiter » à blanc.

- La Compagnie Foncière et Minière, M. Glandu, son directeur, est un proche du Gouverneur. Sa société va se distinguer en utilisant du palissandre comme bois de chauffe et charbon de bois.

La forêt offerte en récompense aux proches du pouvoir va faire des émules. De hauts fonctionnaires coloniaux vont demander et obtenir leur part : Maître Lacaille, avocat et président de la chambre de commerce d'Antananarivo (120 hectares de forêt en 1920), le procureur général Reynaud de Lyques, chef du service judiciaire de la colonie, ou les nombreux colons nécessiteux qui estiment avoir mérité de la patrie.

Jean Fremigacci (1998) juge ainsi cette seconde phase de l'exploitation forestière malgache: "Cette société coloniale va mettre en pièces la forêt malgache, ne pouvant pas concevoir que la production soit autre chose qu'une consommation du capital, dans le cadre d'une pratique qui, plus encore que I'appellation d'économie de pillage, mérite celle d'économie de délinquance: dans le contexte des rapports sociaux coloniaux, le droit est fait pour être violé par les privilégiés (et tel est justement leur privilège) tandis qu'il ne peut être qu'ignoré de la grande masse des sujets. C'est là, déjà, une situation qui annonce l'état patrimonial de l'ère postcoloniale. »

Et aujourd'hui : I'exploitation forestière de Masoala est-elle enfin devenue durable ? Qui sont les exploitants de la forêt de Masoala ? Quelles sont leurs techniques d'extraction, leurs perspectives commerciales et environnementales ? Quel rôle l'État malgache joue-t-il dans cette activité économique? Cette étude a pour objet de faire le point sur ces différents aspects, afin de déterminer ce qui a changé depuis 1850.

\section{MÉTHODE}

Pour établir le profil social des exploitants de bois précieux, nous avons analysé divers documents provenant de l'Administration forestière entre 2009 et 2012. L'essentiel de ces documents concerne la SAVA (Sambava, Antalaha, Vohémar, Andapa), principale région d'extraction des bois précieux à Madagascar. La liste nominative des opérateurs connus officiellement en SAVA au $1^{\text {er }}$ janvier 2012 est donnée en annexe. Nous avons d'autres documents qui concernent les opérateurs de Toamasina, mais ils ont été exclus du cadre de cette étude, car ils ne sont pas aussi complets que ceux de la SAVA. En outre, ils ne portent pas sur plusieurs années. Le rôle de l'État apparaît à travers l'étude de la presse nationale, des jugements de tribunaux et le décalage entre le volume de bois exporté du pays et celui qui est intercepté par les autorités. Quant aux aspects techniques de I'extraction du bois, ils sont connus par les études précédentes (Randriamalala et al. 2010, Stasse, 2002), actualisées par des témoignages oculaires recueillis sur place entre 2009 et 2012.

\section{RÉSULTATS}

Les opérateurs officiels de bois précieux étaient 13 en janvier 2009 (arrêté interministériel n003/2009, signé sous la présidence Ravalomanana). Leur nombre est passé à 15 en juin 2009, puis à 19 en septembre de cette même année. L'arrêté interministériel n³8244/2009 du 21 septembre 2009 autorise en effet une augmentation du nombre d'opérateurs, moyennant une amende à payer par conteneur et une déclaration des stocks détenus (articles 3 et 5). Le nombre d'opérateurs continue d'augmenter pour atteindre 27 en avril 2010, puis 103 pour la seule région SAVA au début de 2012, selon un recensement officiel du Ministère des Eaux et Forêts. Ces chiffres, ainsi que le chronogramme correspondant, sont résumés dans la figure 1.

Il faut noter que l'exportation du bois de rose s'est féminisée au fil du temps, avec l'arrivée dans la filière des épouses, des sœurs ou des filles d'opérateurs. En janvier 2009, les femmes représentaient $15 \%$ des opérateurs, contre $20 \%$ en 2012 (hommes : $66 \%$, sociétés : 14\%). Puis au fil des mois, les opérateurs ont ouvert l'activité au reste de leur famille. Si en 2009, quatre des exploitants avaient des relations familiales 


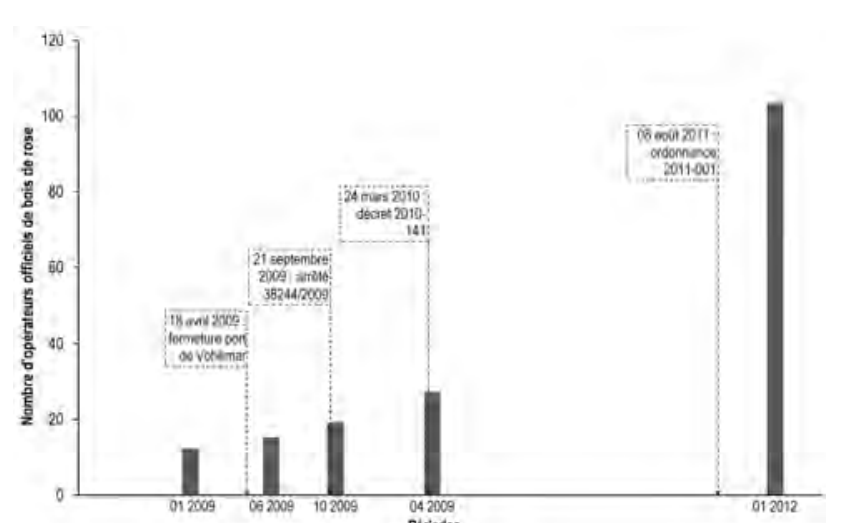

FIGURE 1. Évolution du nombre d'opérateurs en bois précieux, entre janvier 2009 et janvier 2012, inscrits officiellement dans la région SAVA.

(deux fratries), en 2012, ils sont 18 à appartenir à six fratries, trois couples, deux filiations ou à avoir plusieurs de ces liens simultanément.

L'assise géographique de ce commerce s'est également étendue au cours de ces trois années. En 2009, tous les opérateurs étaient originaires d'Antalaha. En 2012, ils proviennent d'Antalaha à $61 \%$, de Sambava à $17 \%$, d'Antananarivo à $7 \%$, de Toamasina et d'Ampanefena (au sud de Vohémar) à $3 \%$ chacune, et de Vohémar à $1 \%$.

Sur le plan judiciaire, dans la liste de janvier 2009, huit personnes avaient déjà comparu devant un tribunal pour exploitation illicite de la forêt et deux avaient été condamnées. Dans la liste de janvier 2012, ces chiffres passent respectivement à 11 et quatre.

Enfin, deux cas individuels sont dignes de mention :

- Malohely Jean-Michel : opérateur historique (il figure dans l'arrêté de janvier 2009), il est le seul à avoir quitté cette activité puisqu'il n'est plus dans la liste des opérateurs de 2012.

- Befototo Angelin : connu officieusement dans ce commerce dès juin 2009 et officiellement en janvier 2012, il est également le leader du parti TGV (parti présidentiel) pour la Région SAVA.

Dans la monographie du Ministère de l'Agriculture, de l'Élevage et de la Pêche (2003) portant sur la SAVA, le recensement général de la population de 1993 évaluait la répartition socioprofessionnelle des chefs de ménage d'Antalaha conformément au Tableau 2, ce qui permet de replacer l'exploitation du bois de rose dans son contexte social et local. À propos des 27000 agriculteurs indépendants de la catégorie $\mathrm{G}$ que ce document recense, voici ce qui est dit (p. 22) : «À la campagne, les paysans - vieillards, femmes, jeunes - pour subsister, pratiquent la vente illicite des bois précieux - bois de rose - bois d'ébène, au prix de : - $\varnothing>20 \mathrm{~cm}$ : 1250 à $1000 \mathrm{Fmg}$ la longueur de $2 \mathrm{~m}$.

$-\varnothing<20 \mathrm{~cm}: 1000 \mathrm{Fmg}$.

Cette pratique vient de l'autorisation de ramassage donnée aux paysans par les autorités au lendemain du passage du cyclone Hudah en avril 2000, car en ce temps-là, beaucoup de ces bois ont été arrachés et charriés par les eaux, la pratique est restée jusqu'à maintenant. »

\section{DISCUSSION}

L'EXPLOITATION DU BOIS DE ROSE, UNE AFFAIRE DE FAMILLE.

L'étude détaillée des liens entre les individus ayant grossi les rangs des exportateurs ou des détenteurs de stocks de bois de rose entre 2009 et 2012 montre une implication progressive des membres des familles du noyau historique. Si au départ un gros exportateur avait sa sœur dans cette activité et un autre y avait son beau-frère, les exportateurs de 2009, constatant d'une part les bénéfices importants de ce commerce, et d'autre part, la relative impunité des participants, ont décidé de faire profiter leur famille de leur savoir-faire. Frères, sœurs, fils, épouses, belles-sœurs ont alors rejoint leurs rangs. Ce choix s'explique de plusieurs façons:

- le savoir-faire et la réputation : les heureux exportateurs de 2009 ont noué des contacts avec les collecteurs qui leur amènent le bois de rose prêt à embarquer: documents d'exploitation en règle, marquage effectué (alors qu'aucun de ces collecteurs n'a de lot d'exploitation forestière : ils achètent le bois aux villageois qui le coupent dans le Parc de Masoala). Dans bien des cas, les exportateurs ne payent pas les collecteurs au comptant. Manquant souvent de liquidités, ils font des dettes qu'ils ne remboursent que Iorsque I'acheteur chinois a payé I'exportation. Pour les collecteurs, c'est donc une affaire de confiance et de réputation. Certains parmi les plus petits d'entre eux n'ont d'ailleurs jamais vu l'argent de leur bois. Ainsi, la réputation d'un exportateur s'étend à sa famille : on peut faire confiance à l'épouse de ..., au frère de ... car l'intéressé est fiable : il paye avec retard, mais il paye toujours. Ce savoir-faire s'étend également aux contacts noués avec l'Administration : à qui et comment demander les agréments d'exportation, comment et à quelle heure franchir les multiples points de contrôle des forces de sécurité, comment procéder avec les Douanes, à quel transporteur confier cette cargaison spéciale, etc. Ce réseau de contacts ne peut profiter qu'à des proches.

- la quantité de bois qui arrive aux exportateurs plus ou moins spontanément est énorme. Elle excède largement ce que l'Administration semble disposée à tolérer : I'arrêté interministériel n`38244/2009 du 21 septembre 2009 limite à 25 conteneurs la quantité de bois possible à l'export pour chaque opérateur (le conteneur, nouvelle unité administrative ?) La solution adoptée par les exportateurs historiques a donc été de grossir leur nombre. Pour ne pas perdre de l'argent en acceptant une concurrence venue d'ailleurs, ils ont mis la famille sur les rangs. Il apparaît ainsi que le commerce du bois de rose est d'abord une aventure individuelle et non une affaire d'entreprise. Nous sommes clairement dans une logique de prédation plus que dans une perspective d'exploitation durable.

- la réputation commerciale doit être sauvegardée et ne peut s'étendre qu'à des proches. Les acheteurs chinois sont en effet méfiants lorsqu'ils viennent prospecter à Madagascar. IIs savent implicitement que tout ce commerce n'est pas légal, sûrement pas durable et qu'il faut faire vite pour s'emparer du maximum de bois disponible avant que l'inévitable pénurie ne fasse grimper les cours à des sommets vertigineux. Ils sont donc prêts à prendre beaucoup de risques et la concurrence entre eux est rude. Certains opérateurs malgaches malveillants en profitent : dans le Marojejy 
en 2009, des collecteurs avaient glissé des pierres dans des billes creuses de bois de rose, alourdissant ainsi le rondin et la facture, mais sans égard pour la scie électrique qui allait le découper en chine ; plus récemment, en mars 2012, un trafiquant de Toamasina a remplacé le contenu de quatre conteneurs de bois de rose par du pin, après le scellement des conteneurs par la Douane et le paiement de la facture par I'acheteur chinois. En mai 2012, Iorsque nous avons été avisés de ces faits, l'acheteur chinois n'a probablement pas encore découvert l'escroquerie. Pour éviter de telles mésaventures dans un commerce par ailleurs assez risqué, la confiance entre acheteur et vendeur est capitale. Cette confiance explique qu'initialement les vendeurs étaient majoritairement des Malgaches d'origine chinoise. Accointances culturelle, linguistique et régionale facilitent cette mise en confiance : beaucoup de vendeurs d'Antalaha et de Toamasina ont leurs racines familiales dans la région de Canton, d'où vient la majorité des acheteurs chinois.

L'EXPLOITATION DU BOIS DE ROSE, UNE AFFAIRE DE VILLAGE.

Si en 2009 les opérateurs étaient tous originaires d'Antalaha, en 2012 cette origine ne représente plus que $61 \%$ du total, soit 63 opérateurs. Cette localisation de la contagion peut s'expliquer de deux façons. La première est bien sûr la proximité de Masoala, lieu de coupe du bois. Le bois est lourd, difficile et dangereux à transporter, il est donc logique de le vendre au port le plus proche, c'est-à-dire Antalaha.

Ensuite, beaucoup d'exploitants historiques sont connus pour leur goût du luxe ostentatoire. Véhicules $4 \times 4$ grand luxe du dernier modèle, avion privé pour aller chercher dans la capitale des maîtresses voyantes, grandes fêtes populaires sur le stade d'Antalaha avec force décibels, bière et zébu à profusion quand il s'agit de mobiliser contre une mesure du gouvernement, une population rurale que I'on amène par camions gratuitement depuis la brousse : les exemples sont légion de la réussite affichée sans complexe par ceux qui se sont lancés dans le commerce du bois de rose.

Vis-à-vis de la population environnante, cet affichage est très efficace. La réussite sociale est manifeste et indiscutable. Elle fait donc des envieux et elle suscite l'émulation. Elle impressionne les fonctionnaires chargés de réprimer ou d'encadrer cette activité. Les voisins, les amis et les membres du réseau des exportateurs voudraient en être aussi. Et ils ont été aidés par les exportateurs historiques pour leurs démarches administratives. D'abord, il y avait suffisamment de bois disponible pour faire de la place à de nouveaux exportateurs. Ensuite, plus le nombre d'exportateurs grandit à Antalaha, moins ceux-ci se sentent marginalisés : I'illégalité devient la norme sociale (rappel : couper, transporter et exporter du bois de rose est illégal depuis le 24 mars 2010). C'est ainsi que sur les 63 opérateurs de 2012 à Antalaha, 40 sont totalement nouveaux dans cette activité : ils ne sont pas d'anciens collecteurs de bois pour les exportateurs historiques, ils ne sont pas de leur famille, ils ne sont pas d'origine chinoise. Ils habitent juste dans la même ville, souvent dans les mêmes quartiers.

Si I'on considère que les exportateurs de bois de rose figurent dans la catégorie $D$ du recensement donné par la Monographie de la SAVA de 2003 (profession intermédiaire, entre profession intellectuelle et scientifique et employé de type administratif), leur nombre actuel représente $19 \%$ de cette catégorie. Même si ce chiffre est à prendre avec précaution en raison de l'âge du recensement (20 ans), son ordre de grandeur est significatif : les exportateurs de bois de rose représentent à Antalaha une minorité numériquement importante, socialement visible, enviée et influente. C'est sans doute de ce rapport de forces sociales qu'ils tirent leur sentiment d'impunité et leur audace de défier publiquement l'État. Ils ont en effet à plusieurs reprises exigé le départ de certains fonctionnaires trop zélés ou des représentants de l'État en charge de réguler le trafic de bois de rose, et ce, avec une totale impunité jusqu'à présent.

Le respect de la loi, en État de droit, suppose l'adhésion du plus grand nombre de citoyens. Les délinquants doivent être minoritaires, numériquement mais aussi socialement et moralement. Lorsque la loi est bafouée par un nombre de personnes tel que la prison devient trop petite ou que la surface sociale des délinquants devient trop importante, alors le système ne peut plus fonctionner et ceux qui respectent la loi se sentent marginalisés. Cette atmosphère délétère pousse un nombre grandissant de fonctionnaires intègres à vouloir quitter Antalaha, où ils sentent au quotidien la réprobation plus ou moins explicite de la population à leur encontre lorsqu'ils accomplissent le travail que leur a confié l'État.

Faire le procès de la pauvreté n'a pas de sens, mais couper des arbrisseaux de moins de vingt centimètres de diamètre n'en a pas non plus. Et la faiblesse de I'Administration, qui tolère qu'à partir de 2001 (Monographie de la SAVA 2003, Stasse 2002), les villageois continuent de couper de jeunes arbres une fois évacués ceux abattus par le cyclone Hudah, ne peut qu'encourager l'émergence d'une nouvelle classe socioprofessionnelle habituée à changer la loi à son profit et à dicter ses règles à I'Administration. À l'instar de la Nation, I'État de droit existe d'abord dans la tête d'une majorité de citoyens : dans la microsociété d'Antalaha, ce n'est plus le cas. Le sens civique a été remplacé par une cupidité contagieuse.

L'EXPLOITATION DU BOIS DE ROSE, UNE AFFAIRE D'ÉTAT ?

Et cette contagion a fini par atteindre Antananarivo, la capitale, puisque sept opérateurs y sont maintenant officiellement déclarés dans les registres de la SAVA. Un seul y était connu dès la fin 2009, les autres sont récents. Quatre d'entre eux agissent en société commerciale et les trois autres en leur nom propre. Comme il n'y a pas de bois de rose à Antananarivo, la seule raison de l'extension de ce commerce à cette ville est, soit la proximité du pouvoir central qui facilite les procédures administratives, soit la capacité d'investir alliée au sens des affaires de certains opérateurs économiques de cette ville. Le commerce du bois de rose n'est plus lié à la seule proximité de la forêt, mais aussi au capital disponible et au pouvoir d'influence.

Mais le plus étrange est sans doute ailleurs. À Madagascar, aucune activité n'a autant défrayé la chronique (Randriamalala et al. 2012) que le trafic de bois de rose depuis le début de la Transition en 2009. Le sujet est devenu d'une extrême sensibilité, la presse nationale en est friande, la communauté internationale le suit de près. Dans ces conditions, comment expliquer l'extraordinaire essor du nombre d'opérateurs officiels (de 19 à 103, soit 542\% d'augmentation entre fin 2009 et début 2012) alors que nous sommes sous un régime clair d'interdiction de l'activité pendant la période considérée ? Le décret n²010-141 (24 mars 2010) interdit toutes les phases de cette activité. L'ordonnance 2011-001 du 8 août 2010 alourdit 
de fortes sanctions pénales le régime d'interdiction en vigueur, mais elle n'empêche pas une augmentation très sensible du nombre d'exploitants officiels. II n'y a que deux explications possibles:

- L'impuissance de l'État : si cette explication est vraie, alors cela signifie que la stratégie d'intimidation et de défi de l'autorité publique menée par les opérateurs de bois de rose a payé. À de nombreuses reprises, les ministres successivement en charge de ce dossier ont conduit des ateliers de "remise à plat », demandé de procéder à un "inventaire général des stocks » et rencontré les opérateurs en colère. Dans cette négociation, les opérateurs ont montré leur détermination plusieurs fois: grandes manifestations publiques antigouvernementales, intimidation des fonctionnaires locaux et régionaux, saccage des locaux des Eaux et Forêts, pillage de dépôts de bois saisis par l'Administration. Pour un régime dont l'assise populaire venait essentiellement des jeunes de la capitale, le risque politique était grand de laisser se développer un sentiment de rejet dans une région enclavée et difficile d'accès, surtout en période de marasme économique, lié à la chute des cours de la vanille autant qu'à la suspension de l'aide internationale. Si de plus, la plupart des procès n'aboutissent pas à une condamnation ferme, soit par influence sur les magistrats, soit par inapplicabilité d'une législation contradictoire, soit à cause des fautes de procédure de l'Administration forestière, alors le sentiment d'impunité s'installe en même temps que s'affiche la réussite sociale des opérateurs. L'augmentation du nombre d'opérateurs ayant déjà eu affaire à la Justice dans la liste 2012 s'explique par le retour dans cette activité d'anciens exploitants déjà connus de la Justice et elle inclut le départ de l'un d'entre eux (Malohely), qui a subi quatre procès entre 2008 et 2009. La simultanéité de I'intimidation de l'État et du sentiment d'impunité est redoutablement efficace.

- La collusion entre l'État et les trafiquants : depuis la suspension de l'aide internationale suite au coup d'État de mars 2009, I'État malgache se trouve en grande difficulté financière. Dans de nombreuses administrations, dont certaines sont primordiales pour la population (santé, éducation, sécurité et ordre public), les budgets de fonctionnement ont été tellement réduits que I'activité a dû baisser elle aussi. Le moral de la population s'en ressent durement et le même processus que celui qui a amené Andry Rajoelina au pouvoir pourrait bien se retourner contre lui : un mouvement de foule organisé en sous-main par des agitateurs expérimentés, qui s'empare des centres de pouvoir après une brève paralysie du pays. La Transition a donc un besoin urgent et vital pour sa survie de trouver des fonds pour faire tourner a minima la machine de l'État. En raison de sa nature même, la Transition est incapable d'avoir une vision politique et économique à moyen terme. L'exploitation des ressources naturelles est donc la meilleure option financière car la plus immédiatement rentable. II est logique que l'État s'entende avec les opérateurs de bois de rose, quitte à violer les lois et règlements qu'il a lui-même édictés, pour ne pas être balayé par un mouvement populaire plus ou moins spontané. L'État a anobli les trafiquants en les faisant opérateurs (Randriamalala 2012), puis il a conclu avec eux un mariage d'intérêt.

Selon le WWF de Madagascar (2012, p. 8) à propos du changement climatique : "Madagascar a été évalué par un bureau international de consultance comme le $3^{\mathrm{e}}$ pays le plus vulnérable au monde après le Bengladesh et l'Inde. » La déforestation sans contrôle qui a lieu depuis 2009 ne peut qu'aggraver cette vulnérabilité.

\section{CONCLUSION}

Les exploitants de bois de rose au début de 2012 sont donc plus nombreux que jamais, sans que l'État ne cherche ou ne parvienne à contenir leur nombre. Ils sont majoritairement originaires d'Antalaha où ils dictent leur loi aux représentants locaux de I'État. Initialement Malgaches d'origine chinoise, ils sont aujourd'hui un peu plus représentatifs de la diversité ethnique nationale. Leur activité a fini par atteindre les milieux d'affaires de la capitale, après s'être étendue aux membres de leurs familles, puis à ceux de leurs réseaux professionnels.

Leur activité est plus cachée des médias qu'elle ne l'est du pouvoir central, avec lequel ils partagent une communauté d'intérêts. L'exploitation de la forêt est conduite dans une perspective exclusivement à court terme, fondée sur I'enrichissement immédiat et la mise à l'abri des capitaux à l'étranger, hors de portée de la Justice malgache.

Par rapport à la période 1850 -1925, évoquée en introduction, certains éléments ont changé :

- Le nombre et la nationalité des opérateurs : de cinq Mauriciens en 1850 à 103 Malgaches en 2012. La localisation des trafiquants est également en train de changer. Avant 2012, ils résidaient tous aux abords de la forêt. Depuis 2012, des opérateurs de la capitale ont rejoint cette activité.

- La quantité de bois extraite annuellement: de 3000 tonnes en 1900 à 52000 tonnes en 2009 ;

- La destination du bois : I'Europe en 1900, la Chine aujourd'hui ; - Le statut de la forêt de Masoala : de domaine royal en 1850 ou colonial après 1896, à Parc National en 2012. En revanche, certains éléments sont invariants :

- Le mode d'exploitation : des investisseurs résidant aux abords de la forêt, qui achètent des billes de bois aux villageois de la forêt, sans se soucier outre mesure de leur origine, de leur diamètre ni de leur nombre. La légalisation a lieu après l'achat. L'exploitation est toujours en mode prédation, avec une préférence marquée pour les zones d'extraction situées à proximité de la côte ou des rivières pour faciliter le transport.

- L'impuissance de l'État au cœur de la forêt : la puissance publique ne peut empêcher la coupe quand elle le décide. Le taux de fraude hors de la forêt (fausses déclarations en Douane) semble à peu près constant, de l'ordre de $50 \%$ pour autant qu'il soit possible d'en juger. Force est de constater que ce qu'écrit Jean Fremigacci (1998) à propos de la première moitié du siècle précédent est toujours vrai : " exploiter la forêt à Madagascar, c'est d'abord exploiter les faiblesses de la réglementation et de l'administration. » 
Que peut-on conclure de ces 160 et quelques années d'observations, qui ne représentent après tout que la moitié de la vie d'un bois de rose ? Que la forêt a été harcelée de multiples manières à un rythme incompatible avec sa régénération naturelle et que rien, dans la conjoncture actuelle, ne permet de prédire une amélioration. Carl von Carlowitz (1713) qui prônait I'exploitation durable de la forêt a sans doute été entendu en Allemagne, puis par contagion en Europe, voire en Chine, au gré des déforestations catastrophiques localement constatées. Au fur et à mesure que ces pays prenaient conscience de l'importance de la préservation de leurs forêts, ils se rabattaient sur des pays qui, noyés dans l'impuissance coloniale ou des crises politiques perpétuelles, étaient moins soucieux de leur avenir, et le sont hélas toujours.

\section{REMERCIEMENTS}

Je tiens à remercier trois rapporteurs anonymes pour leur commentaires constructifs qui ont permis l'amélioration de cette contribution.

\section{RÉFÉRENCES}

Carl von Carlowitz, H. 1713. (Réimprimé en 2009). Sylvicultura oeconomica, oder hausswirthliche Nachricht und Naturmässige Anweisung zur Wilden Baum-Zucht. Verlag Kessel, Remagen-Oberwinter, Germany.

Decary, R. 1962. Lıancien régime de I'or à Madagascar. Bulletin de I'Académie Malgache Nouvelle série 40: 83-95.

Fremigacci, J. 1998. La forêt de Madagascar en situation coloniale: une économie de la délinquance. In: Plantes et Paysages d’Afrique: Une Histoire à Explorer. M. Chastanet (ed.), pp 411-439. Éditions Karthala, Paris.

Ministère de l'Agriculture, de l'Élevage et de la Pêche 2003. Monographie de la Région du SAVA, Ministère de I'Agriculture, de l'Élevage et de la Pêche, Unité de Politique pour le Développement Rural, juin 2003.

Petit, M. et Jacob, G. 1964. Un essai de colonisation dans la baie d'Antongil, (1885-vers 1926). Annales de I'Université de Madagascar, Lettres 4: 33-56.

Randriamalala, H. 2012. La bolabolacratie. tribune.com <http://www.madagascar-tribune.com/La-bolabolacratie,17164.html> téléchargé le $1^{\text {er }}$ mars 2012

Randriamalala, H. \& Liu, Z. 2010. Rosewood of Madagascar: Between democracy and conservation. Madagascar Conservation \& Development 5 , 1: 11-22.

Randriamalala, H., Waeber, P. et Wilmé, L. 2012. Les cyber-verts contre le trafic de bois de rose malgache. In: Madagascar, Le Coup d'État de Mars 2009. S. Randrianja (ed.), pp 123-145. Éditions Karthala, Paris.

Stasse, A. 2002. La Filière Bois de Rose. Région d'Antalaha - Nord-est de Madagascar. Thèse de mastère non publiée, Université de Montpellier, Montpellier, France.

WWF MWIOPO 2012.Changement Climatique: Les principaux engagements de la COP 17. Bulletin d'information avril 2012. <http://awsassets. panda.org/downloads/newsbites2012q1_fr_lr.pdf> téléchargé le $1^{\text {e }}$ juillet 2012.

\section{MATÉRIEL SUPPLÉMENTAIRE.}

DISPONIBLE EN LIGNE UNIQUEMENT.

TABLEAU S1. Origine socioprofessionnelle des 103 opérateurs de bois précieux connus dans la région SAVA en janvier 2012.

TABLEAU S2. Répartition socioprofessionnelle des chefs de ménage d'Antalaha (extrait du Recensement général de la population de 1993 In Ministère de l'Agriculture, de I'Élevage et de la Pêche 2003)

TABLEAU S3. Liste des opérateurs de bois de rose connus dans la région SAVA en janvier 2012. 